\title{
A zonal model for assessing the infection risk distribution of COVID-19 in indoor environments
}

\author{
Marco Marigo ${ }^{1, *}$, Giacomo Tognon ${ }^{1}$, Michele De Carli ${ }^{1}$ and Angelo Zarrella ${ }^{1}$ \\ ${ }^{1}$ Department of Industrial Engineering, University of Padova, 35131 via Venezia 1, Padova, Italy
}

\begin{abstract}
Nowadays, the search for new solutions to the pandemic situation caused by the spread of SARS-CoV-2 is one of the most important issues worldwide. The difficulties encountered in finding an effective cure for the infection and the ongoing vaccination campaign place a priority on minimising the transmission of this disease. This paper deals exclusively with the airborne route of transmission for COVID-19, proposing a model for the evaluation of infection risk.

It starts from the well-known Wells-Riley model and its successive modifications and tries to couple it with a zonal model which analyses the air movement in an indoor environment. The objective of this model is evaluating whether the infection risk depends considerably on the position of the infected subject and the susceptible person in the room and how different ventilation concepts affect these aspects.

The model was applied to an office room with a mixing ventilation system. The results obtained concern the sub-division of the considered volume in four cells, each one perfectly mixed. The evaluation of infection risk in different positions was carried out and an analysis on the parameters affecting this value was performed.
\end{abstract}

\section{Introduction}

Buildings are one of the main energy consumption sectors. Taking into consideration both residential and commercial ones, they were responsible for about the $40 \%$ of the final energy use in Europe in 2018, with a consequent noticeable environmental impact in terms of greenhouse gas emissions [1].

Since people spend most of their time indoors, the achievement of a good indoor environmental quality (IEQ) is fundamental to keep their satisfaction level high. HVAC system operation is required to warrant thermal comfort and suitable indoor air quality (IAQ). The former is maintained through heating and cooling plant, whereas the latter involves the supply of outdoor air by ventilation.

Indoor Air Quality is the indicator of the salubrity of the indoor environment, since it basically depends on the presence of pollutants in the air. The topic has sparked great interest with the beginning of the ongoing COVID-19 pandemic, caused by the spread of the SARS-CoV-2, whose existence was first reported to the WHO Country Office in China

* Corresponding author: marco.marigo@unipd.it 
on $31^{\text {st }}$ December 2019 [2]. According to the scientific community, the transmission of this respiratory disease is caused by contact with the infected source or contaminated surfaces (fomites), and by large expired droplets [3]. The inhalation of airborne microdroplets, called droplet nuclei, is a third route of infection, but there is still great debate on its relevance. However, the documented outbreaks at a restaurant in Guangzhou, China, and at a choir rehearsal in Mount Vernon, Skagit County (USA) seem to prove the possibility of the airborne transmission in indoor environments [4-5].

The airborne infection route is the result of subsequent phases, namely generation of virus-laden droplet nuclei, air transport, inhalation by the exposed person [6-7]. The infected source releases droplets through common respiratory activities such as normal breathing, speaking, coughing, sneezing. After exhalation, they undergo a rapid evaporation, due to environmental temperature and relative humidity. Some of them fall by gravity before evaporating within a distance of $1 \div 2 \mathrm{~m}$, whereas the others become droplet nuclei and remain suspended in the air for prolonged times and distances. A conventional limit diameter of $5 \mu \mathrm{m}$ is usually considered to discern between droplets and droplet nuclei [8]. The transport phase defines the spread of pathogens and their concentration at the breathing zone of an individual. Inhalation determines the intake dose of the susceptible subject and his infection risk.

The spread of the pandemic places a priority in investigating how ventilation could be effective in minimising the concentration of pathogens in the indoor air, making the environment healthier. Many researchers have pursued this objective by means of experimental studies or CFD analyses. CFD models allow to characterise the fluid flow, by the numerical resolution of the Navier-Stokes equations [9]. In one study, a CFD model was built to verify the suitability of displacement ventilation systems in hospital rooms with bedded patients, at different ventilation rates $\left(6 \mathrm{~h}^{-1}, 9 \mathrm{~h}^{-1}, 12 \mathrm{~h}^{-1}\right)$. The authors concluded that pathogens concentration and infection risk were maximum at the breathing zone of a standing healthcare worker, discouraging the employment of this ventilation method in healthcare facilities [10]. Another study assesses the risk of airborne influenza transmission in bus microenvironment, with different ventilation rates and concepts. The obtained results suggest that displacement ventilation defines suitable airflow patterns for the removal of pathogens close to the infected source, lowering the infection risk [11]. CFD models are the best way to observe the actual spatial distribution of airborne pathogens, but they present some drawbacks: they are time expensive and require high computational resources for the simulations.

Some simple mathematical models have been developed to describe the infection risk for susceptible people, according to how much infectious material they inhale. They can be divided into two categories: those derived from Wells-Riley equation and dose-response models [12]. The former are based on the concept of quantum, a hypothetical dose unit which makes the $63.2 \%$ of an exposed population sick, as stated in the original definition given by Wells [13]. They consider the indoor space as perfectly mixed and calculate the infection risk through an exponential probability equation [14-15]. Despite their simplicity, they return a uniform infection risk within the space, regardless of the distance between the infected source and the susceptible subject.

In this paper, a model for assessing the infection risk from COVID-19 in indoor environments is presented. It originates from the need to reach a compromise between the well-mixed space assumption and the computational effort of CFD analyses. The idea is the macro-discretisation of the interested domain into different cells to evaluate the infection risk in each zone [16]. The aim of the model is observing the link between the infection risk and the relative position between infected source and susceptible person. It has been applied to two case studies regarding a single office room of $4 \times 4 \times 2.7 \mathrm{~m}$, and an open 
space office of $8 \times 8 \times 2.7 \mathrm{~m}$, to investigate the importance of parameters such as ventilation rate, room volume and use of masks.

\section{Methodology}

In this section the development of a risk zonal model is discussed. The principle is the modification of the well-mixed room assumption to give a certain spatial resolution to the infection risk within the environment. The overall model is obtained coupling a ventilation zonal model to the infection probability calculation. The former involves a discretisation of the geometrical domain into multiple zones to define the interzonal airflows between adjacent cells transporting infectious material. The second step of the model is the calculation of the airborne infection risk for each zone. Pathogen concentration within each zone is evaluated through a balance equation, where the airflows calculated in the first part, are integrated. Finally, a risk equation is applied.

\subsection{Ventilation zonal model: POMA}

The first part of the model consists of a ventilation zonal model, built for the definition of air movements within the interested space. In this work the zonal model called POMA (Pressurized ZOnal Model with Air Diffuser) was applied [17][18]. Zonal method is an intermediate approach between nodal modelling and CFD simulations [19]. The principle of the zonal modelling is the macro-discretisation of the analysed room into different cells or zones, each one well-mixed, as shown in Figure 1.

This model is developed to deal with mechanically ventilated spaces, and it is founded on some basic assumptions. The discretisation grid is composed of parallelepiped-shaped cells, therefore only vertical and horizontal boundaries are present. Moreover, each zone is well-mixed in terms of air temperature and density, whereas the pressure is hydrostatically distributed, starting from a reference value at the bottom level. Thus, the pressure profile is characterised by a decrease along $z$ coordinate (see the coordinate system in Figure 1).
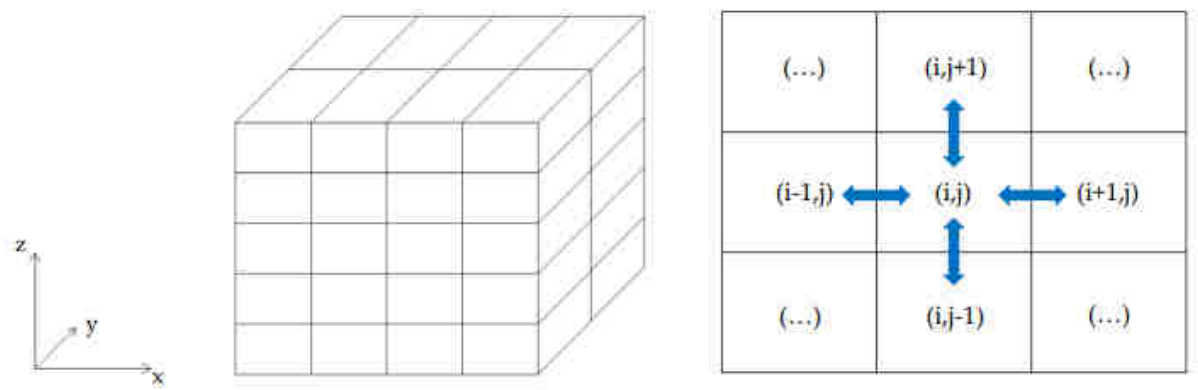

Fig. 1. Zonal modelling approach. Example of a 3D discretisation and partition shown on a plane with interactions between cells.

The thermodynamic properties of each zone are linked by the ideal gas law:

$$
P_{\text {ref }} /=R_{\text {air }} \cdot T
$$

where $R_{\text {air }}$ is the gas constant for air, equal to $287 \mathrm{~J} \mathrm{~kg}^{-1} \mathrm{~K}^{-1}$.

Regions affected by particular airflows such as thermal plumes of the internal heat sources and boundary layers at the walls are not modelled. Air jets are not modelled spatially, but the supply and exhaust airflows are respectively considered as input and 
output terms in the mass balance of single zones. Therefore, each cell is surrounded by two types of boundary surfaces: normal interfaces of separation with adjacent zones, and, for the outer zones, wall boundaries which are portions of wall interested only by heat exchanges. The mass and heat flows are modelled for each boundary.

\subsubsection{Interzonal flowrates modelling}

Concerning the interzonal mass flowrates between adjacent zones, they are calculated as a function of the pressure difference across the normal boundary, through the well-known Power Law:

$$
m=\cdot k \cdot A \cdot \Delta P^{n}
$$

Parameters $k$ and $n$ are the flow coefficient (i.e., permeability of the boundary) and the flow exponent, respectively. For $k$, a value of $0.83 \mathrm{~m} \mathrm{~s}^{-1} \mathrm{~Pa}^{-\mathrm{n}}$ is taken from previous studies, while for $n$ a value of 0.5 is assigned considering turbulent airflow [17]. Equation (2) takes different shapes depending on the orientation of the boundary.

Vertical boundaries are characterised by a variable pressure difference along the surface due to the linear distributions of pressure at both sides. The situation is shown in Figure 2. At a certain height $z$, these profiles may intersect, leading to a null pressure difference. This point is called neutral plane $Z_{n}$, and there is no horizontal airflow across it. The height of this plane is determined as follows:

$$
Z_{n}=\Delta P_{r e f} /(g \cdot \Delta)
$$

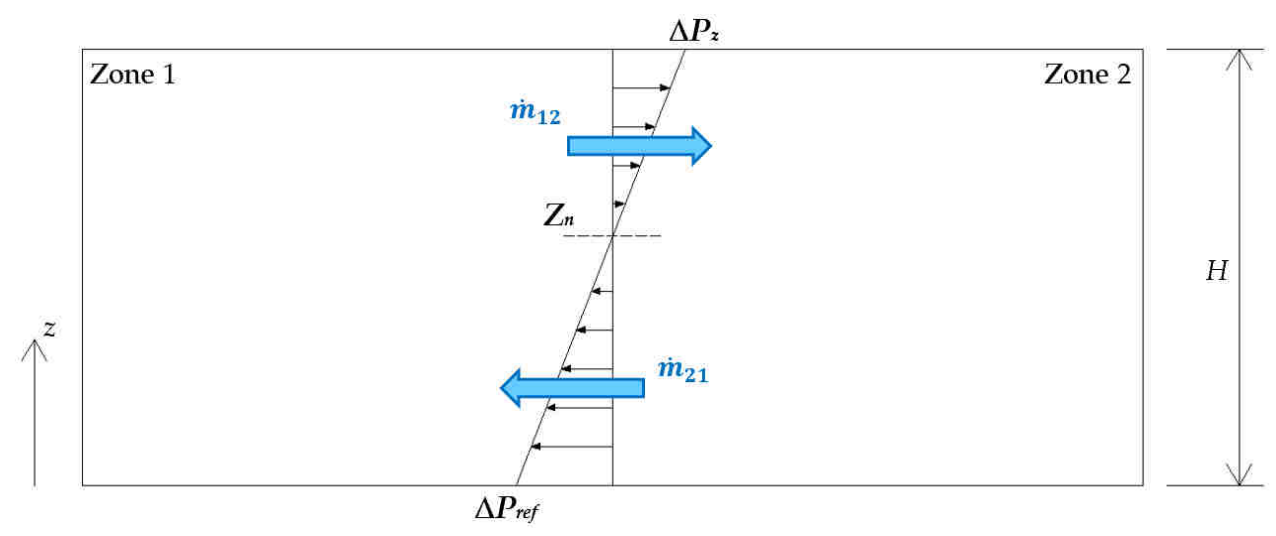

Fig. 2. Vertical normal boundary modelling.

On the basis of the neutral plane position, the mass flowrates above and below it are calculated integrating the Power Law (Equation (2)) along $z$ coordinate:

$$
\begin{array}{r}
m o-Z n=0-Z n \cdot k \cdot L \cdot|\Delta \cdot g|^{n} \cdot\left|Z Z_{n}\right|^{n+1} /(n+1) \\
m Z n-H=Z n-H \cdot k \cdot L \cdot|\Delta \cdot g|^{n} \cdot|Z n-H|^{n+1} /(n+1)
\end{array}
$$

$L$ is the depth of the vertical interface. $m o-Z n$ is the mass flowrate crossing the interface portion from 0 to $Z_{n}$, whereas $m Z n-H$ is that crossing the section from $Z_{n}$ to $H$.

Conversely, for the horizontal boundaries, the reference pressure of the upper zone must be compared to the pressure at the summit of the lower one, leading to a constant pressure difference along the interface. 


\subsubsection{Heat flows modelling}

As regards the energy transfers, radiative heat exchanges and solar radiation are neglected. The only heat flows are those related to the enthalpy carried by the interzonal mass flowrates, and the heat exchange with the outdoor environment through the building envelope by conduction and convection.

The first ones are modelled as follows, considering the interzonal mass flowrate from the $j$-th zone to the $i$-th one and the temperature difference between these adjacent cells

$$
q_{h, j i}=m_{j i} \cdot c_{p, a i r} \cdot\left(T_{j}-T_{i}\right)
$$

It is assumed dry air, so $c_{p, \text { air }}$ is equal to $1005 \mathrm{~J} \mathrm{~kg}^{-1} \mathrm{~K}^{-1}$.

The transmission through the envelope structures of zone $i$ can be easily computed

$$
q_{\text {h,transm }, i}=U \cdot A_{\text {wall }} \cdot\left(T_{i}-T_{\text {air }, \text { ext }}\right)
$$

$U$ is the thermal transmittance of the building structure and $T_{\text {air }}$ ext is the external air temperature.

\subsubsection{Equation system and model resolution}

For each zone i, along with the ideal gas law, mass and energy balance equations are written under steady-state conditions

$$
\begin{aligned}
& m_{j i}-\quad m_{i j}+m_{\text {sup }}-m_{\text {rem }}=0 \\
& q_{h, j i}-q_{h, \text { transm }, i}+q_{h, \text { sup }}-q_{h, \text { rem }}=0
\end{aligned}
$$

The system has $3 n_{z o n}$ equations and $3 n_{z o n}$ unknown variables (i.e., $T$, , and $P_{r e f}$ for each zone), where $n_{z o n}$ is the number of cells. However, only $n_{z o n}-1$ mass balances are linearly independent. Therefore, a reference pressure of one zone is fixed as parameter to make the system resolvable, with $3 n_{z o n}-1$ equations and $3 n_{z o n}-1$ unknown properties. Once the system is solved, the interzonal mass flowrates can be explicitly computed reapplying Power Law equations. Finally, the volumetric flow rates are easily determined.

\subsection{Infection risk model}

The second step of the model deals with the airborne infection risk calculation within each cell. The quantity of airborne pathogens is defined in terms of quanta.

The infected source releases quanta into the indoor environment through exhalation at a rate that is specific for the considered disease. Indoor air becomes the carrier for the spread of these fictitious particles across the space. A quanta balance equation is set for each $i$-th cell in order to obtain the zonal concertation of airborne material

$$
V_{i} \cdot d C_{i} / d t=q_{i} \cdot I_{i}-Q_{o i} \cdot C_{i}-Q_{i j} \cdot C_{i}+Q_{j i} \cdot C_{j}
$$

Where $C$ is quanta concentration, $q$ is quanta generation rate for the disease, $I$ is the number of infected people in the zone, $Q_{o}$ is the exhaust air flow rate. The terms $Q_{i j}$ and $Q_{j i}$ represent the interzonal flow rates calculated from the zonal model.

Fresh air from diffusers is not considered in the balance, since it is assumed that outdoor air does not contain infectious quanta.

Quanta concentrations represent the infectious material available at the breathing zone of a susceptible subject. In accordance with the formulation given by Wells [12], the zonal 
infection risk $P_{I, i}$ for the susceptible individual is finally calculated by an exponential probability equation

$$
P_{I, i}=\left[1-\exp \left(-p \cdot \int_{t, \exp } C_{i}(t) d t\right)\right] \cdot 100
$$

Where $p$ is the breathing flow rate and $t_{\exp }$ is the exposure time interval. The exponential term in Equation (11) represents the intake dose of the susceptible subject.

The overall zonal risk model has been built in MATLAB environment.

\section{Model application and simulations}

The model has been used to simulate real case studies, for the calculation of the airborne infection risk from COVID-19 in ventilated indoor spaces. It was firstly applied to a typical single office room and then to an open space office room with a fourfold volume compared to the previous one. The objective is the analysis of the influence of different parameters such as the relative position of the infected source and susceptible person, ventilation rate (Air Change per Hour, $A C H$ ), room volume, on the risk extent. Moreover, the employment of masks has been included.

\subsection{Geometrical domain and zonal discretisation}

The first case-study concerns a single office room with dimensions of $4 \times 4 \times 2.7 \mathrm{~m}$ and a volume of $43.2 \mathrm{~m}^{3}$, as shown in Figure 3. It is based on the "CORE-CARE Laboratory" at the Industrial Engineering Department of Padova, which is a climate chamber for carrying out analyses on indoor environmental quality with radiant systems.

The outdoor air is supplied through a mixing ventilation system, with two linear diffusers at the top of the North wall and two exhaust grilles at the bottom of the South wall. The East wall constitutes the external façade of the room, whereas the others are internal partitions.

The geometrical domain has been divided into four identical well-mixed cells with a cross section on the plan view (Figure 3).

The second case-study deals with an open space office room with a fourfold volume, i.e., $172.8 \mathrm{~m}^{3}$. The discretisation grid is identical to the previous case, and dimensions have been chosen so that a single zone corresponds exactly to the original office room in terms of volume, as indicated by Figure 4 .
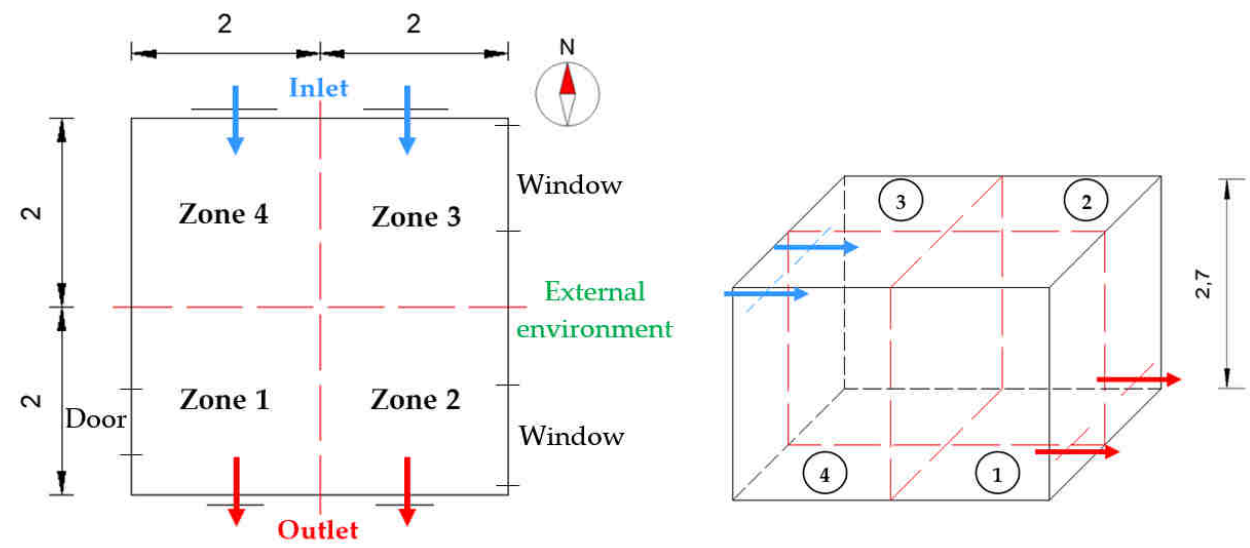

Fig. 3. Dimensions and discretisation grid of the single office room. 


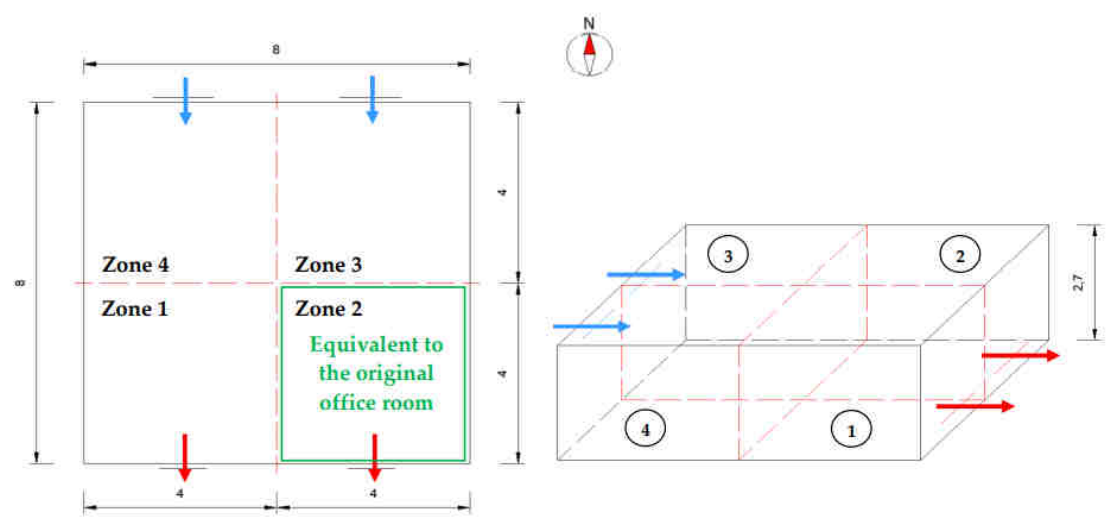

Fig. 4. Dimensions and discretisation grid of the open space office room.

\subsection{Assumptions and simulation parameters}

As the zonal model is concerned, some assumptions about mass and heat transfers between cells were done: no air infiltrations (i.e., airtight room); no solar radiation; only one external wall, the others are considered as adiabatic; no internal heat gains.

Table 1 shows the set simulation parameters for the calculation of the interzonal flow rates. The reference pressure of Zone 1 is fixed as parameter to substitute one of the linearly dependent mass balances. The external air temperature is set to $-5^{\circ} \mathrm{C}$. Finally, fresh air is supplied at $20^{\circ} \mathrm{C}$.

Table 1. Fixed parameters for the zonal model.

\begin{tabular}{|c|c|c|c|}
\hline \multicolumn{2}{|c|}{ Parameter } & \multicolumn{2}{c|}{ Fixed Value } \\
\hline Reference pressure of Zone 1 & $P_{\text {ref, },}$ & 101325 & $\mathrm{~Pa}$ \\
\hline External air temperature & $T_{\text {air }, \text { ext }}$ & -5 & ${ }^{\circ} \mathrm{C}$ \\
\hline Air jet temperature & $T_{\text {jet }}$ & 20 & ${ }^{\circ} \mathrm{C}$ \\
\hline Air jet density & $\rho_{\text {jet }}$ & 1.204 & $\mathrm{~kg} \mathrm{~m}^{-3}$ \\
\hline East wall thermal transmittance & $U$ & 0.3 & $\mathrm{~W} \mathrm{~m}^{-2} \mathrm{~K}^{-1}$ \\
\hline
\end{tabular}

Each linear diffuser contributes to half of the total flow rate. Similarly, the same flowrate is extracted equally divided into the two exhaust grilles.

The infection risk assessment has been carried out considering one infected source and one susceptible person sharing the room. For the sake of simplicity, the infected source stands in Zone 1, whereas the zonal risk is evaluated for each other position. The subjects perform typical office tasks; subsequently, the applied breathing flowrate $p$ and quanta emission rate $q$ are based on a sedentary activity level. For COVID-19 a quanta generation rate of 20 quanta $\mathrm{h}^{-1}$ has been taken from a recent paper [20]. Finally, for the susceptible person an exposure time of $8 \mathrm{~h}$ is considered, i.e., a typical workday without breaks. These parameters are reported in Table 2. 
Table 2. Simulation parameters for the infection risk assessment.

\begin{tabular}{|c|c|c|c|}
\hline \multicolumn{2}{|c|}{ Parameter } & \multicolumn{2}{c|}{ Fixed Value } \\
\hline Breathing flowrate & $p$ & 0.54 & $\mathrm{~m}^{3} \mathrm{~h}^{-1}$ \\
\hline Quanta emission rate for COVID-19 & $q$ & 20 & quanta $^{-1}$ \\
\hline Exposure time & $t_{\exp }$ & 8 & $\mathrm{~h}$ \\
\hline
\end{tabular}

A sensitivity analysis has been carried out to observe the influence of different parameters on infection risk extent. These parameters are:

- position of the susceptible individual;

- ventilation rates $A C H$ of $1,1.5$, and $2 \mathrm{~h}^{-1}$;

- use of surgical masks and FFP2 respirators;

- room volume $V$ of 43.2 and $172.8 \mathrm{~m}^{3}$.

Equations (10) and (11) have been solved under transient conditions.

\subsection{Use of personal protective devices}

The filtration effect offered by surgical and FFP2 masks has been analysed. Protection devices are characterised by two parameters: filtration efficiency $X$ and leakage factor $Y$ due to poor fit. The global filtration efficiency $Z$ in percentage is given by

$$
Z=X-X \cdot Y / 100
$$

The properties of surgical masks and FFP2 respirators are reported in Table 3, distinguishing exhalation (ext) from inhalation (int) flows.

Table 3. Properties of surgical and FFP2 masks.

\begin{tabular}{|c|c|c|c|}
\hline \multirow{2}{*}{ Type of protection } & \multicolumn{2}{|c|}{ Filtration efficiency [\%] } & Leakage factor [\%] \\
\cline { 2 - 4 } & $\boldsymbol{X}_{\text {int }}$ & $\boldsymbol{X}_{\text {ext }}$ & $\boldsymbol{Y}$ \\
\hline Surgical mask & 20 & 95 & 27 \\
\hline FFP2 & 94 & 94 & 11 \\
\hline
\end{tabular}

The properties of FFP2 are taken from European Standard [21]. On the other hand, Standard EN 14683 gives the filtration efficiency of surgical masks only for exhalation [22]. A low $X_{\text {int }}$ value has been chosen due to the inefficiency of surgical masks in protecting the wearer, but ad-hoc test would be necessary to confirm this aspect. Finally, the leakage factor $Y$ has been deduced from tests carried out by some researchers [23].

In the analysed situations, both the infected source and the susceptible person wear the same type of protection. Therefore, masks have a filtration effect both on exhalation and inhalation of quanta, as expressed by Equations (13) and (14).

$$
\begin{aligned}
& q^{\prime}=q \cdot\left(1-Z_{\text {ext }} / 100\right) \\
& p^{\prime}=p \cdot\left(1-Z_{\text {int }} / 100\right)
\end{aligned}
$$

Where $q$ ' and $p$ ' are the reduced quanta generation rate and the reduced breathing flow rate, respectively. 


\section{Results}

The results obtained from the simulations of the analysed case studies reveal the influence of the interested parameters on the infection risk.

\subsection{Position of the susceptible person}

The adopted discretisation grid basically defines a well-mixed environment. Therefore, the final infection risk is almost uniform within the space, as shown in Figure 5.

This outcome highlights that the relative position between susceptible and infected source has a limited effect. However, this aspect is reasonable considering that for a such small volume, the indoor environment easily reaches a perfect mixing.

From infection risk values reported in Table 4, it can be noticed that the spatial distribution of risk is slightly more heterogeneous for higher ventilation rates.

Table 4. Zonal infection probabilities after $8 \mathrm{~h}$.

\begin{tabular}{|c|c|c|c|c|}
\hline \multirow{2}{*}{ ACH $\left[\mathbf{h}^{-1}\right]$} & \multicolumn{4}{|c|}{ Zonal infection probability $\boldsymbol{P}_{\boldsymbol{I}}[\%]$ after $\mathbf{8 ~ h}$} \\
\cline { 2 - 5 } & Zone 1 & Zone 2 & Zone 3 & Zone 4 \\
\hline 1.0 & 83.36 & 82.08 & 80.84 & 81.28 \\
\hline 1.5 & 71.71 & 69.57 & 67.47 & 68.19 \\
\hline 2.0 & 62.34 & 59.52 & 56.74 & 57.68 \\
\hline
\end{tabular}

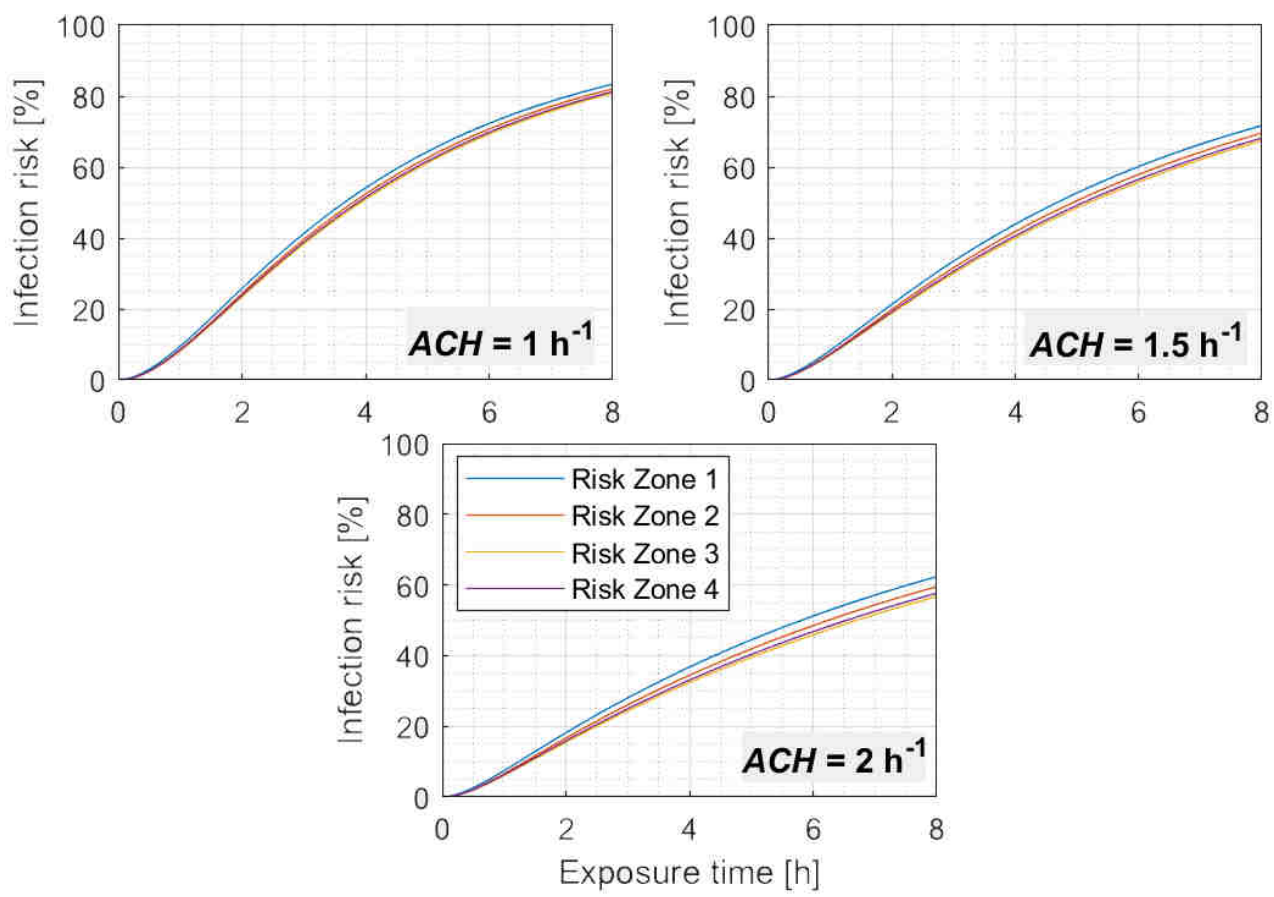

Fig. 5. Spatial distribution of infection risk. 


\subsection{Effect of ventilation rate}

Increasing the air exchange rate $(A C H)$ promotes dilution and subsequent removal of pathogens, reducing the infection risk and proving to be an effective mitigation strategy against airborne transmission. Figure 6 highlights risk trends for the considered ventilation rates. The infection risk goes from $80 \%$ to $60 \%$ if the ventilation rate is increased from $1 \mathrm{~h}^{-1}$ to $2 \mathrm{~h}^{-1}$. The effect of this parameter can be also observed from Table 4 .
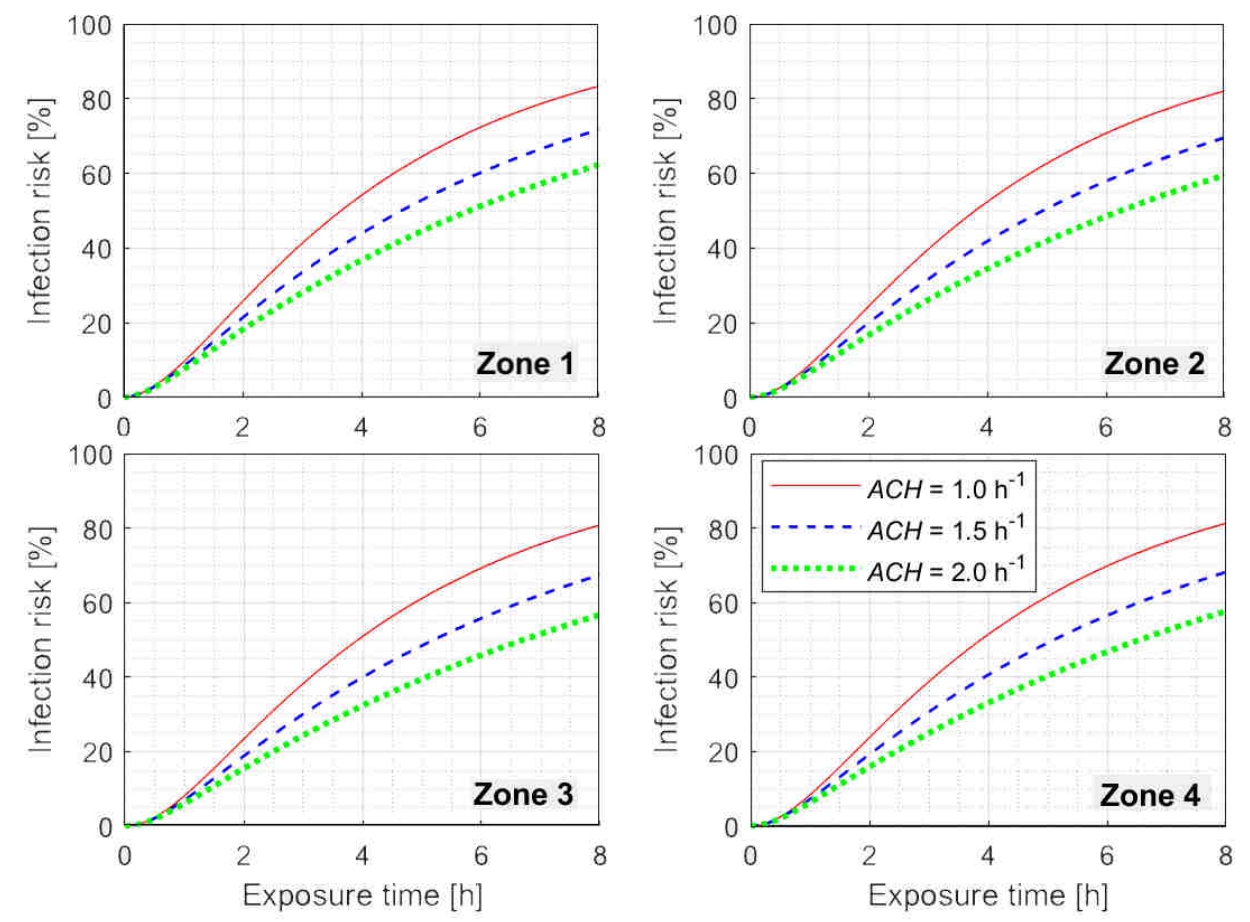

Fig. 6. Influence of $\mathrm{ACH}$ on infection risk.

\subsection{Wearing masks}

The filtration effect of masks is clearly underlined in Figure 7 for the case with a ventilation rate of $1 \mathrm{~h}^{-1}$. FFP2 respirators offer a higher protection level than surgical masks. Wearing FFP2 respirators means keeping infection risk below 5\%, which can be considered an acceptable threshold level.

\subsection{Effect of room volume}

Larger volume entails a higher dilution of airborne infectious material and a corresponding lower risk, with a reduction of about 50\%, as clearly shown in Figure 8 for the case with $1 \mathrm{~h}^{-1}$ and no masks. For the open space office, interestingly, higher relative deviations between zonal infection probabilities can be observed compared to the first case, i.e., a more heterogeneous spatial risk is obtained. This means that, for the larger room, the zonal approach seems to be more effective. Table 5 depicts this aspect for $A C H=1 \mathrm{~h}^{-1}$. 
Table 5. Effect of volume on spatial distribution of risk.

\begin{tabular}{|c|c|c|c|c|c|}
\hline \multirow{2}{*}{$\mathbf{A C H}\left[\mathbf{h}^{-\mathbf{1}}\right]$} & \multirow{2}{*}{$\mathbf{V}\left[\mathbf{m}^{\mathbf{3}}\right]$} & \multicolumn{4}{|c|}{ Zonal infection probability $\boldsymbol{P}_{\boldsymbol{I}}[\%]$ after $\mathbf{8 ~ h}$} \\
\cline { 3 - 6 } & & Zone 1 & Zone 2 & Zone 3 & Zone 4 \\
\hline \multirow{2}{*}{$\mathbf{1 . 0}$} & 43.2 & 83.36 & 82.08 & 80.84 & 81.28 \\
\cline { 2 - 6 } & 172.8 & 36.78 & 34.46 & 32.34 & 33.08 \\
\hline \multirow{2}{*}{$\begin{array}{c}\Delta \boldsymbol{P}_{\mathbf{I}, \%}[\%] \\
\text { vs Zone 1 }\end{array}$} & 43.2 & 0 & -1.54 & -3.02 & -2.50 \\
\cline { 2 - 6 } & 172.8 & 0 & -6.31 & -12.07 & -10.06 \\
\hline
\end{tabular}
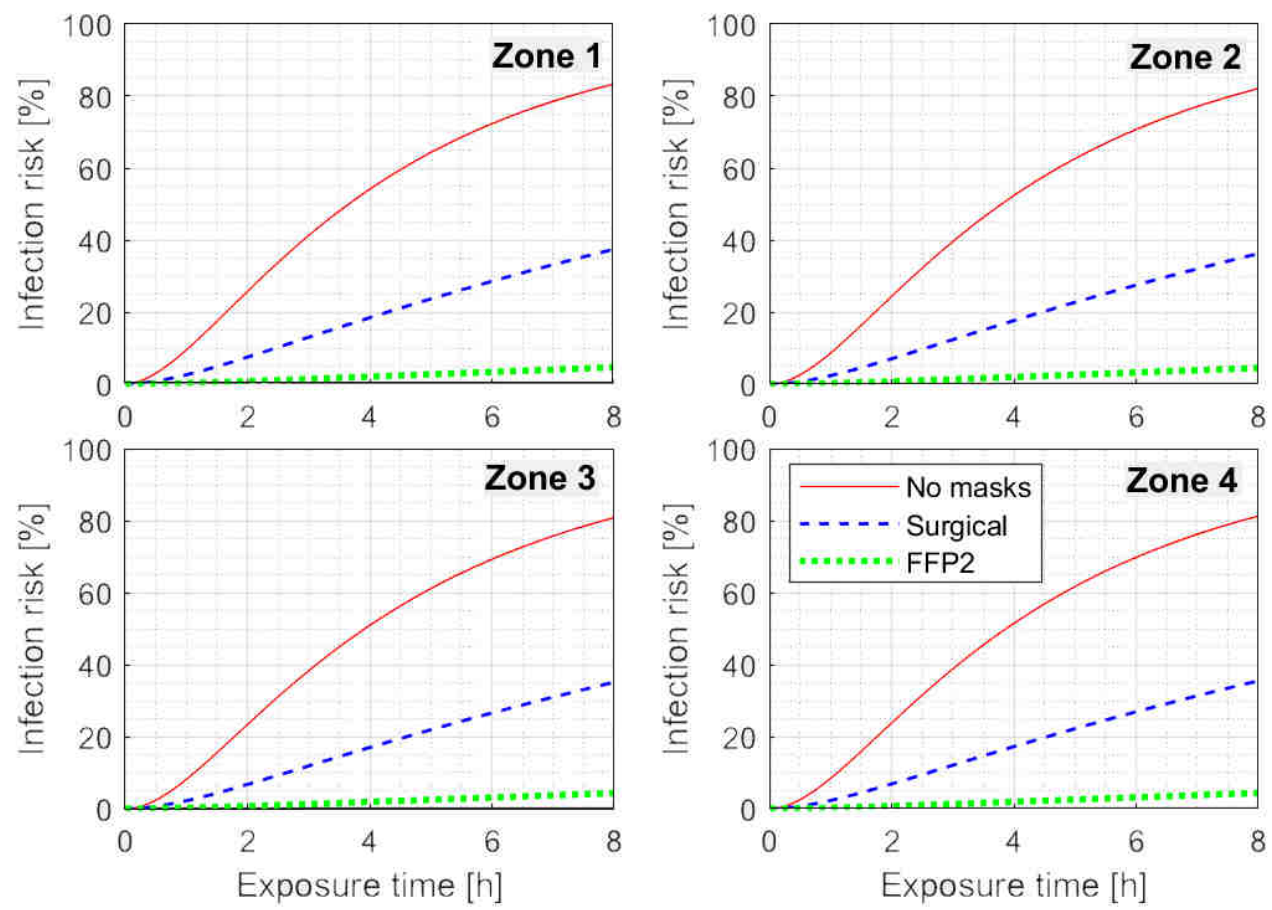

Fig. 7. Influence of mask use on infection risk. 

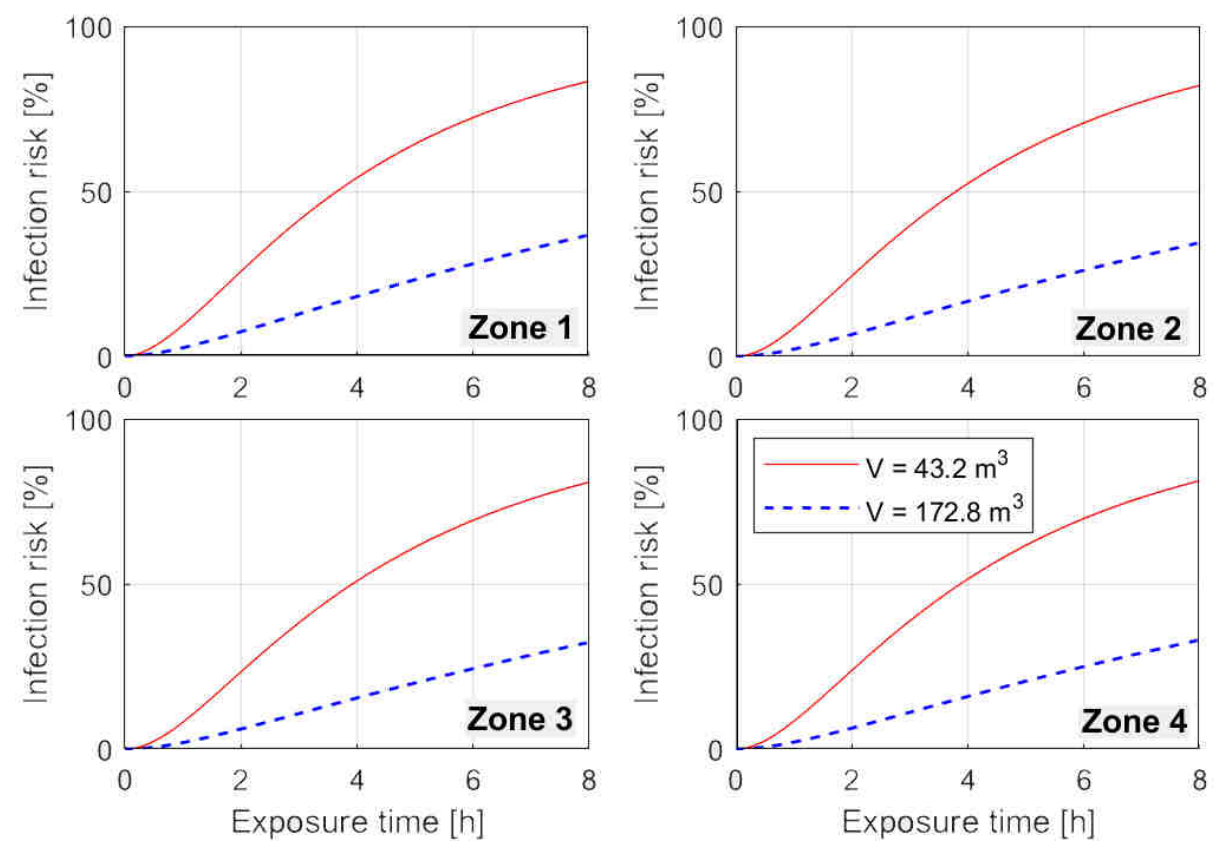

Fig. 8. Influence of volume on infection risk.

\section{Conclusions}

In this paper the problem of airborne transmission of COVID-19 in indoor environments has been taken on. A zonal risk model has been developed to evaluate the spatial distribution of the infection risk within the space.

The model has been applied with a four-cells grid to a typical single office room, with dimensions of $4 \times 4 \times 2.7 \mathrm{~m}$, and to an open space office, characterised by a fourfold volume compared to the first case study. The influence of different parameters such as position of the susceptible person, ventilation rate, room volume and filtration efficiency of masks, has been investigated.

The adopted grid basically defines a well-mixed environment, with an almost uniform infection risk across the space. Therefore, the relative position between susceptible person and infected source has a limited effect. In the case of the open space office, the infection risk is slightly more heterogeneous than in the smaller office room.

Higher ventilation rates $(A C H)$ promote the dilution and removal of airborne pathogens, leading to lower infection probabilities. Similarly, an enhanced dilution is observed with larger room volume, with a subsequent positive effect on risk.

A substantial reduction of the infection risk is achieved through the employment of personal protection devices. Wearing FFP2 respirators enables to keep the infection probability under a level of $5 \%$.

In conclusion, the developed model is a starting point for future more complete risk assessments. Some limitations emerge from simplifications and assumptions adopted for its definition, and future improvements are necessary to guarantee more reliable, consistent results, including a denser discretisation grid to investigate the imperfect mixing of the space and the effect of thermal stratification due to buoyancy.

\section{Symbology}




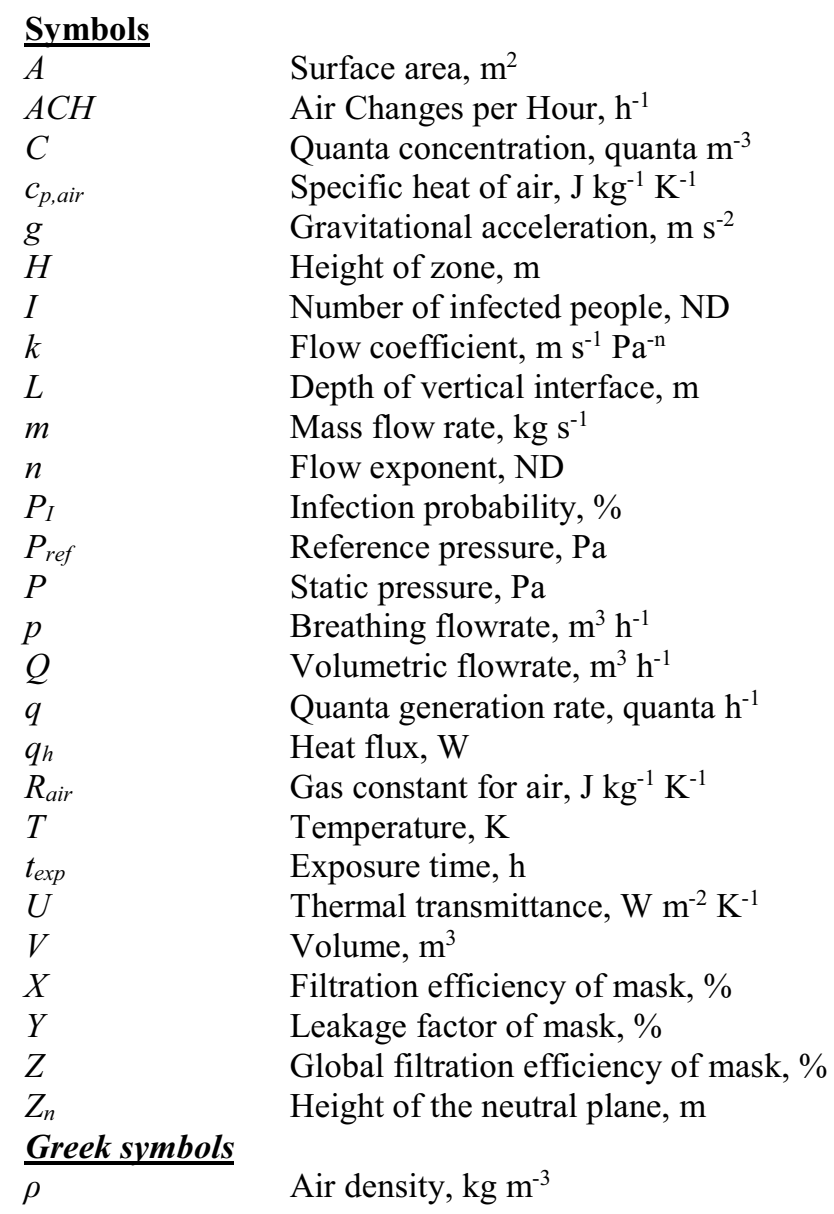

\section{References}

[1] Eurostat, Energy data 2020 edition, (2020).

[2] L. Morawska, J. Cao, Airborne transmission of SARS-CoV-2: The world should face the reality, Environ. Int., 139, 105730 (2020).

[3] L. Morawska, J.W. Tang, W. Bahnfleth, P.M. Bluyssen, A. Boerstra, G. Buonanno, J. Cao, S. Dancer, A. Floto, F. Franchimon, C. Haworth, J. Hogeling, C. Isaxon, J.L. Jimenez, J. Kurnitski, Y. Li, M. Loomans, G. Marks, L.C. Marr, L. Mazzarella, A.K. Melikov, S. Miller, D.K. Milton, W. Nazaroff, P. V. Nielsen, C. Noakes, J. Peccia, X. Querol, C. Sekhar, O. Seppänen, S. ichi Tanabe, R. Tellier, K.W. Tham, P. Wargocki, A. Wierzbicka, M. Yao, How can airborne transmission of COVID19 indoors be minimised?, Environ. Int., 142 (2020).

[4] G. Buonanno, L. Stabile, L. Morawska, Estimation of airborne viral emission: Quanta emission rate of SARS-CoV-2 for infection risk assessment, Environ. Int. 141, 105794 (2020). 
[5] S.L. Miller, W.W. Nazaroff, J.L. Jimenez, A. Boerstra, G. Buonanno, S.J. Dancer, J. Kurnitski, L.C. Marr, L. Morawska, C. Noakes, Transmission of SARS-CoV-2 by inhalation of respiratory aerosol in the Skagit Valley Chorale superspreading event, Indoor Air, 31, 314-323 (2021).

[6] A.A. Aliabadi, S.N. Rogak, K.H. Bartlett, S.I. Green, Preventing Airborne Disease Transmission: Review of Methods for Ventilation Design in Health Care Facilities, Adv. Prev. Med., 2011, 1-21 (2011).

[7] J. Wei, Y. Li, Airborne spread of infectious agents in the indoor environment, Am. J. Infect. Control., 44, S102-S108 (2016).

[8] E.Y.C. Shiu, N.H.L. Leung, B.J. Cowling, Controversy around airborne versus droplet transmission of respiratory viruses: implication for infection prevention, Curr. Opin. Infect. Dis., 32, 372-379 (2019).

[9] Z.T. Ai, A.K. Melikov, Airborne spread of expiratory droplet nuclei between the occupants of indoor environments: A review, Indoor Air, 28, 500-524 (2018).

[10] J.M. Villafruela, I. Olmedo, F.A. Berlanga, M. Ruiz de Adana, Assessment of displacement ventilation systems in airborne infection risk in hospital rooms, PLoS One, 14 1-18 (2019).

[11] S. Zhu, J. Srebric, J.D. Spengler, P. Demokritou, An advanced numerical model for the assessment of airborne transmission of influenza in bus microenvironments, Build. Environ., 47, 67-75 (2012).

[12] G.N. Sze To, C.Y.H. Chao, Review and comparison between the Wells-Riley and dose-response approaches to risk assessment of infectious respiratory diseases, Indoor Air, 20, 2-16 (2010).

[13] E.A. Nardell, Wells Revisited: Infectious Particles vs. Quanta of Mycobacterium tuberculosis Infection-Don't Get Them Confused, Mycobact. Dis., 06, 8-11 (2016).

[14] L. Gammaitoni, M.C. Nucci, Using a Mathematical Model to Evaluate the Efficacy of TB Control Measures, Emerg. Infect. Dis., 3, 335-342 (1997).

[15] S.N. Rudnick, D.K. Milton, Risk of indoor airborne infection transmission estimated from carbon dioxide concentration, Indoor Air, 13, 237-245 (2003).

[16] C.J. Noakes, P. Andrew Sleigh, Mathematical models for assessing the role of airflow on the risk of airborne infection in hospital wards, J. R. Soc. Interface., 6, (2009).

[17] F. Haghighat, Y. Li, A.C. Megri, Development and validation of a zonal model POMA, Build. Environ., 36, 1039-1047 (2001). 
[18] Y. Lin, POMA - A Zonal Model for Airflow and Temperature Distribution Analysis, Concordia University Montreal, Quebec, Canada, 1999.

[19] Y. Lu, J. Dong, J. Liu, Zonal modelling for thermal and energy performance of large space buildings: A review, Renew. Sustain. Energy Rev., 133, 110241 (2020).

[20] G. Buonanno, L. Morawska, L. Stabile, Quantitative assessment of the risk of airborne transmission of SARS-CoV-2 infection: Prospective and retrospective applications, Environ. Int., 145, 106112 (2020).

[21] European Committee for Standardization-CEN, EN 149:2009, Respiratory protective devices - Filtering half masks to protect against particles - Requirements, testing, marking, CEN, Brussels, Belgium, 2009.

[22] European Committee for Standardization-CEN, EN 14683:2019, Medical face masks - Requirements and test methods, CEN, Brussels, Belgium, 2009.

[23] A. V. Mueller, M.J. Eden, J.M. Oakes, C. Bellini, L.A. Fernandez, Quantitative Method for Comparative Assessment of Particle Removal Efficiency of Fabric Masks as Alternatives to Standard Surgical Masks for PPE, Matter, 3, 950-962 (2020). 